\title{
As A Clinical and Radiographic Syndrome Posterior Reversible Encephalopathy Syndrome (PRES)
}

\section{Sonmezler A* \\ Department of Neurology, Adana City Research and Training Hospital, Turkey}

*Corresponding author: Abdurrahman Sonmezler, Department of Neurology, Adana City Research and Training Hospital, Adana, Turkey; Email: sonmezlera@gmail.com

\section{Mini Review \\ Volume 4 Issue 3}

Received Date: September 24, 2019

Published Date: October 01, 2019

DOI: $10.23880 /$ accmj-16000160

\section{Abstract}

PRES is a clinic radiological syndrome characterized by neurologic findings with edema predominantly in the posterior region of the brain. Pathophysiology is not clear but the final problem is thought to be cerebral vasogenic edema. It usually occurs with seizures, unconsciousness, headache, visual abnormalities, nausea / vomiting and more rarely with hemiparesis or aphasia. MRI is vital for diagnosis. MRI findings are characterized by hyper intense signals in fluidattenuated inversion recovery sequences .PRES is usually reversible due to vasogenic edema. Therefore, PRES has a relatively good prognosis. MRI findings and clinical symptoms are reversible, especially if the syndrome is treated early and appropriately.

Keywords: Reversible Encephalopathy; Cerebral vasogenic edema; Neurologic findings; Magnetic resonance imaging

Abbreviations: PRES: Posterior reversible encephalopathy syndrome; MRI: Magnetic resonance imaging; CT: Computed tomography; FLAIR: Fuidattenuated inversion recovery; ADC Apparent diffusion coefficient; DWI: Diffusion-weighted images.

\section{Introduction}

Posterior reversible encephalopathy syndrome (PRES) is a clinical and radiological description presented in 1996 by Hinchey et al. Posterior reversible leukoencephalopathy syndrome is the most commonly used name. It often presents with seizures, disorders of consciousness, headache, visual abnormalities, nausea/vomiting and more rarely hemiparesis or aphasia. The diagnosis of this syndrome is made by the presence of bilateral, symmetrical cerebral edema, which is more prominent in the parietal and occipital region on magnetic resonance imaging (MRI) along with clinical findings [1].

Vasogenic edema occurs predominantly in the parietooccipital region, but lesions affecting areas such as the frontal lobe, cerebellum, or basal ganglia are also seen. It is thought that the posterior region is prone to be affected because the control of the sympathetic nervous system is weaker in the vertebrobasilar system than in the internal 


\section{Anaesthesia \& Critical Care Medicine Journal}

carotid artery system. The pathogenesis of the syndrome is unclear. However, brain auto regulation disorder and endothelial dysfunction processes are the underlying mechanisms. Increased cerebral vasodilastation causes increased permeability of the blood brain barrier and the passage of fluid and blood products to the cerebral parenchyma. Reactive cerebral vasoconstriction secondary to increased cerebral vasodilation leads to local hypo perfusion, cytotic edema and cerebral ischemia. Increased vascular wall permeability and increased permeability in the blood brain barrier cause fluid and blood products to pass into the cerebral parenchyma. Although the pathophysiological mechanism is associated with different causes, the final problem is thought to be cerebral vasogenic edema. In a nutshell different hypotheses have been reported; cerebral vasoconstriction, which may cause infarcts in the brain, disruption of cerebral auto regulation leading to vasogenic edema, and endothelial damage leading to disruption of blood and brain barrier that leads to accumulation of fluid and protein in the brain.

PRES is a reversible pathogenesis due to vasogenic edema; however, some patients later experience brain infarction and cerebral hemorrhage due to brain cell and endothelial cell death. Various factors that may cause PRES include hypertensive encephalopathy, eclampsia, collagen disease and severe infection and hemolytic uremic syndrome and thrombotic thrombocytopenic purpura. Certain drugs, including immune suppressants, anticancer drugs, and antivirals may be causal agents [24].

PRES is considered to be a differential diagnosis in various neurological, psychiatric, and ophthalmological disorders. Signs and symptoms of PRES are not specific. The diagnosis of PRES is often made after excluding other mimickers like posterior circulation stroke, cerebral venous thrombosis, and leukoaraiosis. The most common neuroimaging abnormality in PRES includes edema without infarction involving the subcortical white matter in the posterior cerebral hemispheres. Calcarin and paramedian occipital lobe structures are usually preserved; it is a condition that separates PRES from bilateral posterior cerebral artery infarction. Although pathology may be seen on computed tomography (CT), CT is not successful in definitive diagnosis and differential diagnosis. Therefore, the diagnostic imaging method in PRES is MRI. MRI is used to perform neuroimaging and T2-weighted or FLAIR (fluid-attenuated inversion recovery) sequences are sensitive to show hyperintense lesions. The typical imaging findings of PRES are most apparent as hyper intensity on FLAIR images. MRI findings are characterized by hyper intense signals in FLAIR (fluid-attenuated inversion recovery) and ADC (apparent diffusion coefficient) without DWI (diffusionweighted images). MRI is vital for diagnosis. However, the diagnosis of PRES is not always possible with MRI findings and clinical evaluation is important for a definitive diagnosis [5].

Differential diagnosis of PRES is infectious encephalitis, autoimmune encephalitis, malignancy, cerebral vascular disease cerebral ischemia, cerebral venous sinus thrombosis, toxic leukoencephalopathy, osmotic demyelination syndrome, metabolic encephalopathy [6]. Although reversible by definition, secondary complications such as ischemic infarction or intracranial hemorrhage have been reported. Most patients recover completely within a few days with early recognition and immediate treatment of the underlying cause. Main treatment is the removal of the underlying cause but lowering of blood pressure is mandatory in cases with severe hypertension. Mortality and morbidity in PRES depends on the underlying disease, for example in eclampsia patients women with PRES have a better prognosis than chemotherapy-associated PRES patients [6].

Case reports with PRES are increasing day by day. PRES is a clinic radiological syndrome characterized by neurologic findings with edema predominantly in the posterior region of the brain. PRES has been increasingly recognized in the last two decades, but with its broad clinical and radiological features, many aspects of this syndrome are still unclear. Although there is still a need to develop and confirm diagnostic criteria and clinical algorithms to standardize the diagnosis of PRES, it is important to recognize patients early and be aware of the need for prompt treatment.

\section{References}

1. Hinchey J, Chaves C, Appignani B, Breen J, Pao L, et al. (1996) A reversible posterior leukoencephalopathy syndrome. N Engl J Med 334 (8): 494-500.

2. Sudulagunta SR, Sodalagunta MB, Kumbhat $M$, Settikere Nataraju A (2017) Posterior reversible encephalopathy syndrome (PRES). Oxf Med Case Reports 2017(4): 43-46. 


\section{Anaesthesia \& Critical Care Medicine Journal}

3. Bartynski WS (2008) Posterior reversible encephalopathy syndrome, part 1: fundamental imaging and clinical features. AJNR Am J Neuroradiol 29(6): 1036-1042.

4. Bartynski WS (2008) Posterior reversible encephalopathy syndrome, part 2: controversies surrounding pathophysiology of vasogenic edema. AJNR Am J Neuroradiol 29(6): 1043-1049.
5. Tetsuka S, Ogawa T (2019) Posterior reversible encephalopathy syndrome: A review with emphasis on neuroimaging characteristics. J Neurol Sci 404: 7279.

6. Lamy C, Oppenheim C, Mas JL (2014) Posterior reversible encephalopathy syndrome. Handb Clin Neurol 121: 1687-1701. 